\title{
Effets d'une sécheresse édaphique sur le fonctionnement hydrique d'Abies bornmulleriana en conditions naturelles
}

\author{
A Granier*, F Colin \\ avec la collaboration technique de $B$ Clerc et $F$ Willm
}

INRA, centre de recherches de Nancy, Champenoux, 54280 Seichamps, France

(reçu le 31 octobre 1989 ; accepté le 25 janvier 1990)

\begin{abstract}
Résumé - L'effet d'une contrainte hydrique estivale a été étudié sur des sapins (Abies bornmulleriana Matt) adultes en conditions naturelles, grâce à un dispositif de type lysimétrique à fond non clos (fig 1). Le potentiel hydrique de base des arbres du traitement sec a montré une décroissance continue, pour atteindre une valeur proche de $-1,2 \mathrm{MPa}$ en fin d'expérimentation, alors que le potentiel hydrique de milieu de journee ne diminuait que faiblement. La transpiration des arbres a été évaluée à partir des mesures de flux de sève brute dans le tronc ; la transpiration relative des arbres du traitement sec par rapport aux témoins bien arrosés a diminué jusqu'à atteindre 0,25 au plus fort de la sécheresse. Un abaissement relatif des conductances stomatiques a aussi été observé. Après réhumectation, $20 \mathrm{j}$ environ ont été nécessaires pour que les transpirations reviennent a leur niveau initial. Les relations journalières entre densité de flux de sève et potentiel hydrique foliaire ont servi à évaluer pour chaque arbre la conductance hydraulique spécifique $C$ (voir équation (2)) du sol à la feuille. Ce paramètre a lui aussi montré une forte diminution lorsque la sécheresse s'est accrue : $C$ passe de $0,26510^{-5}$ a $0,10510^{-5} \mathrm{~mol} \cdot \mathrm{m}^{-2} \cdot \mathrm{s}^{-1} \cdot \mathrm{Pa}^{-1}$ pour des potentiels de base de -0.53 et $-1.17 \mathrm{MPa}$ respectivement. Enfin, une partie de la variabilité des paramètres hydriques a été mise en relation avec le statut concurrentiel des arbres dans le peuplement.
\end{abstract}

transpiration / conductance stomatique / conductance hydraulique / sécheresse / potentiel hydrique

Summary - Effects of soil drought on water relations in Ables bornmulleriana under natural conditions. The effect of soil drying during the summer was studied in situ on adult firs (Abies bornmulleriana Matt) submitted to an artificial drought by the suppression of incident rainfall and lateral ground water infiltration. The predawn xylem water potential of stressed trees showed a continuous decrease down to $-1.2 \mathrm{MPa}$ at the end of the experiment, while the mid-day water potential showed a slight decrease. Tree transpiration was evaluated by sap flow measurements in the trunks. The transpiration ratio of stressed trees versus irrigated ones decreased to a minimum value of 0.25 , while stomatal conductances were reduced. After rehydration, the stressed trees took 20 to recover potential transpiration. Hydraulic conductance

\footnotetext{
* Correspondance et tirés à part
} 
from the soil to twigs $(C)$ was calculated from diurnal relationships between sap flux density and xylem water potential (see eq 2). Hydraulic conductance showed a large decrease as drought increased : $C$ was reduced from $0.26510^{-5} \mathrm{~mol} \cdot \mathrm{m}^{-2} \cdot \mathrm{s}^{-1} \cdot \mathrm{Pa}^{-1}$ to 0.105 $10^{-5} \mathrm{~mol} \cdot \mathrm{m}^{-2} \cdot \mathrm{s}^{-1} \cdot \mathrm{Pa}^{-1}$. During the same period, the predawn water potential decrease from $-0.53 \mathrm{MPa}$ to $-1.17 \mathrm{MPa}$. Between-tree variability of water relations was explained by the crown status of the trees.

transplration / stomatal conductance / hydraullc conductance / drought / water potential

\section{INTRODUCTION}

En France, le sapin pectiné (Abies alba) peut présenter, même dans son aire naturelle, des limitations de croissance, voire des dépérissement (Levy et Becker, 1987) lors des années exceptionnellement sèches. L'extension de cette espèce à des zones à plus forte probabilité de sécheresse peut présenter des risques importants.

Le recours à des espèces proches et moins sensibles aux contraintes hydriques peut constituer une solution de remplacement. Parmi ces espèces, Abies bornmulleriana Matt originaire du Nord de la Turquie comme Abies nordmanniana auquel il est apparenté pourrait présenter des capacités de résistance à la sécheresse intéressantes.

L'objectif de cette étude est de vérifier cette hypothèse et, à cet effet, un ensemble de méthodes écophysiologiques a été mis en œuvre sur des arbres en conditions naturelles pour déterminer l'effet d'une contrainte hydrique estivale sur les relations hydriques de cette espèce.

\section{MATÉRIEL ET MÉTHODES}

\section{Site expérimental}

L'expérimentation a été réalisée dans l'arboretum d'Amance, près de Nancy $\left(48^{\circ} 44^{\prime} \mathrm{N}\right.$ $6^{\circ} 14^{\prime} \mathrm{E}$, altitude $250 \mathrm{~m}$ ), dans une plantation d'Abies bornmulleriana Matt ágée de 25 ans. Le sol est de type brun faiblement lessivé, développé sur des marnes. Les horizons superficieis sont essentiellement limono-argileux, et au-dessous de $50 \mathrm{~cm}$ environ apparaît un horizon de pseudogley.

La placette étudiée comporte 50 arbres plantés à l'écartement de $2 \times 2 \mathrm{~m}$ et les individus les plus grands mesurent entre 11 et $12 \mathrm{~m}$ de hauteur. La circonférence moyenne était de $41 \mathrm{~cm}$, au début des mesures et l'indice foliaire de la placette a été évalué à 7 (Colin, 1988).

Les arbres dominants du placeau ont un houppier très pyramidal, tandis que les individus dominés sont plus fusiformes. Les rameaux sont porteurs de 7 années d'aiguilles au maximum.

Quatre arbres voisins ont été choisis pour constituer le traitement sec, grâce à un dispositif permettant de supprimer les apports d'eau par les précipitations et d'eliminer les transferts hydriques latéraux dans le sol (fig 1). Il s'agit en fait d'un lysimètre construit in situ, mais dont le fond n'est pas clos ; I'horizon de pseudogley limite les transferts d'eau dans le sens vertical. La toiture est constituée de plaques de fibre de verre et résine et les murs latéraux sont en béton hydrofugé doublé de 2 feuilles de polyéthylène, enfoncés à $1,20 \mathrm{~m}$ de profondeur dans le sol. Ce lysimetre délimite une surface au sol de $16 \mathrm{~m}^{2}$ environ.

Trois arbres, hors de ce dispositif, ont été choisis pour servir de témoins bien alimentés en eau, par irrigation manuelle lors des périodes sèches.

Le tableau I reporte les principales caractéristiques des 7 arbres qui composent le dispositif. La surface foliaire des arbres a été estimée au moyen de régressions effectuées entre le diametre de 70 branches prélevées sur 6 arbres et la masse foliaire supportée par chaque branche (poids sec), et des régressions entre le poids sec foliaire et la surface projetée des aiguilles (planimètre Delta-T devices). 


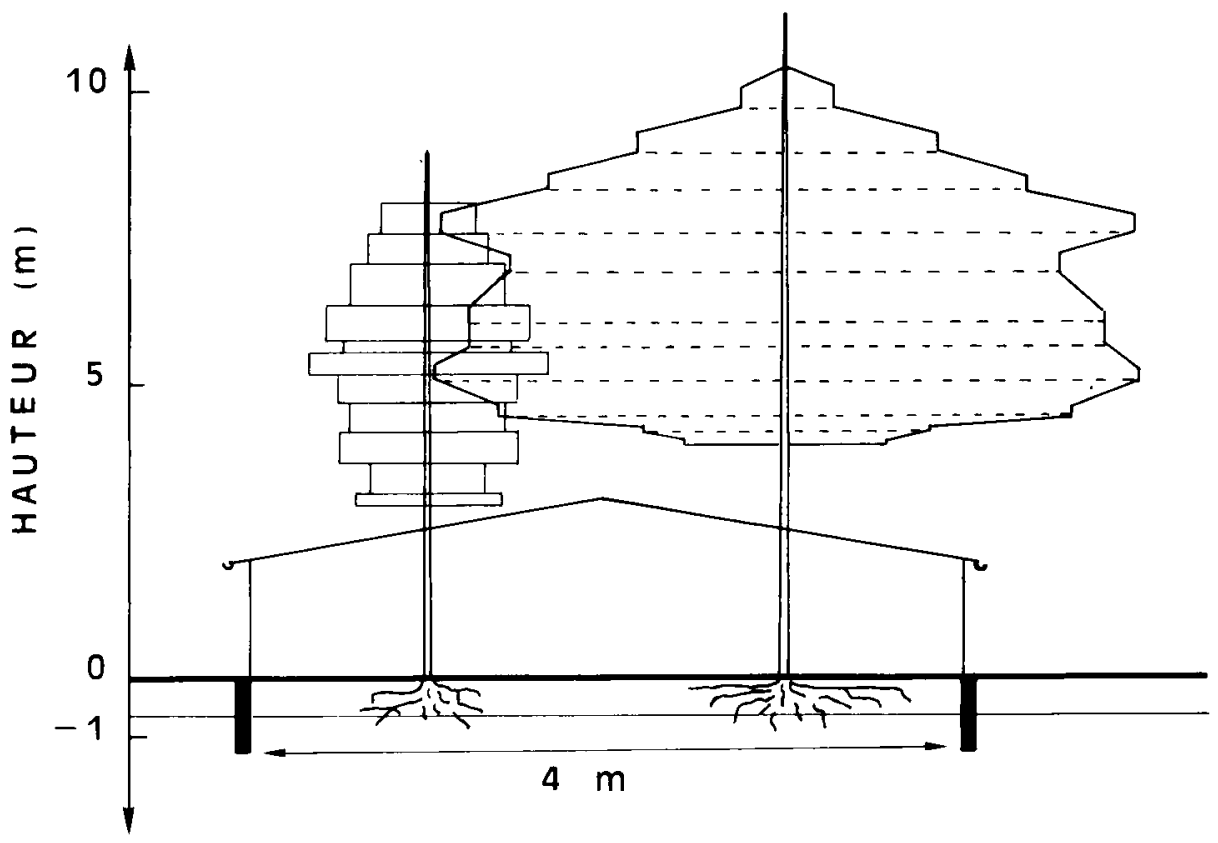

Fig 1 Schéma en coupe du dispositif expérimental utilisé pour le contrôle de la sécheresse.

Tableau I. Caractéristiques des 7 arbres étudiés.

\begin{tabular}{lcccccc}
\hline $\begin{array}{c}\text { Traite- } \\
\text { ment }\end{array}$ & $\begin{array}{c}\text { Arbre } \\
\left(n^{\circ}\right)\end{array}$ & $\begin{array}{c}\text { Hauteur } \\
(m)\end{array}$ & $\begin{array}{c}\text { Circonference } \\
(\mathrm{cm}) \text { a } 1,30 \mathrm{~m}\end{array}$ & $\begin{array}{c}\text { Surface } \\
\text { foliaire }\left(\mathrm{m}^{2}\right)\end{array}$ & $\begin{array}{c}\text { Section } \\
\text { aubier }\left(\mathrm{cm}^{2}\right)\end{array}$ & $\begin{array}{c}\text { Rang } \\
\text { social }\end{array}$ \\
\hline Sec & 4 & 9,6 & 38,4 & 18,5 & 80,3 & dominé \\
& 5 & 8,5 & 29,6 & 14,7 & 44,7 & dominé \\
& 14 & 11,9 & 53,0 & 50,2 & 165,2 & dominant \\
& 15 & 10,9 & 55,8 & 37,8 & 187,5 & dominant \\
Humide & 19 & 9,5 & 41,5 & - & 97,2 & codominant \\
& 28 & 10,6 & 40,0 & 30,4 & 83,9 & codominant \\
& 29 & 11,9 & 58,6 & 62,5 & 206,4 & dominant \\
\hline
\end{tabular}

\section{Méthodes d'étude}

Le flux de sève a été mesuré sur chacun des 7 arbres au moyen de fluxmètres thermiques (Granier, 1985, 1987) positionnés dans le tronc a $1,50 \mathrm{~m}$ de hauteur environ. Ce système permet de mesurer une densité de flux de sève (df, en $\mathrm{dm}^{3} \cdot \mathrm{dm}^{-2} \cdot \mathrm{h}^{-1}$ ) moyenne selon l'axe radial dans le bois d'aubier du tronc. Le calcul du flux total ( $F$ en $\left.\mathrm{dm}^{3} \cdot \mathrm{h}^{-1}\right)$ nécessite la connaissance de la section transversale de bois d'aubier (SA en $\mathrm{dm}^{2}$ ) au niveau de la mesure :

$$
F=d f S A
$$


Une grande partie des résultats a été exprimée en densité de flux, ce qui permet de comparer le fonctionnement d'arbres de dimensions différentes. En effet, on sait (Grier et Waring, 1974 ; Whitehead, 1978) que des arbres en peuplement fermé présentent une relation linéaire entre la section transversale de bois d'aubier (à un niveau de référence, souvent $1,30 \mathrm{~m}$ ) et leur surface foliaire. De ce fait, les densités de flux de sève et de flux en phase vapeur, cumulees sur la journée, sont proportionnelles, si l'on néglige les phénomenes de stockage d'eau dans l'arbre entre le tronc et la feuille.

Le potentiel hydrique $(\Psi f)$ a été mesuré au moyen d'une chambre à pression, sur des rameaux prélevés systématiquement à deux niveaux de hauteur dans chaque arbre : dans la partie supérieure de la couronne, sur des branches de pleine lumiere, et dans la moitié inférieure, sur des branches situees le plus souvent à l'ombre.

La conductance stomatique a été évaluée à l'aide d'un autoporometre (Li-Cor 1600 ) sur des rameaux porteurs d'aiguilles âgées de 1 et 2 ans ; un coefficient de pondération établi statistiquement (Colin, 1988) a permis de corriger ces mesures en fonction de la représentativité des 2 dernières années d'aiguilles par rapport à l'ensemble des aiguilles d'une branche ou d'un verticille. La conductance moyenne a été calculée à partir de 20 mesures réparties sur les 2 mémes niveaux de hauteur que les mesures de potentiel hydrique.

La conductance hydraulique spécifique ( $C$ en $\mathrm{mol} \cdot \mathrm{m}^{-2} \cdot \mathrm{s}^{-1} \mathrm{~Pa}^{-1}$ ) a été calculée pour les 7 arbres du dispositif, lors de journées ensoleillées, à partir des relations entre le potentiel hydrique des rameaux (moyenne des 2 niveaux de hauteur), et la densité de flux de sève circulant au même moment dans le tronc. Ces relations sont en theorie (Landsberg et al, 1976) lineaires et on peut écrire :

$$
\Psi b-\Psi f=1 / C d f
$$

Ainsi la pente de la relation $\Psi f(d f)$ est égale à $-1 / C$ et l'ordonnée à l'origine à $\Psi b$ (potentiel hydrique de base, a flux nul) :

Dans la réalité, la non linéarité des cinétiques diurnes, généralement observées (Schulze et al, 1985 ; Granier et al, 1988) indique un régime de flux hydrique non conservatif, notamment entre le niveau de mesure du flux de sève brute et les ra- meaux, mais on négligera ce phénomène en supposant que l'eau perdue le matin par les tissus de l'arbre est regagnée presque totalement dans la deuxieme partie de la journée par ces mêmes tissus.

Un poste météorologique automatique a été installé au niveau supérieur des couronnes, et a permis de mesurer les principaux paramètres climatiques pendant la duree de l'expérimentation : rayonnement global et net, température, tension de vapeur d'eau, vent.

\section{Déroulement des mesures}

Le toit du lysimètre a été installé au début du mois de mai, alors que le sol présentait un excès d'eau, que l'on a laissé se résorber. Les mesures ont débuté le 21 juin 1988 et se sont achevées le 21 septembre. La saisie des données des flux de sève et des paramètres climatiques a été effectuée au moyen d'une centrale de mesures (Campbelle Ltd CR 7), avec un intervalle de $10 \mathrm{~s}$ entre les scrutations et stockage des moyennes horaires.

Le potentiel de base a été mesuré systématiquement chaque semaine sur les 7 arbres étudiés. Pendant la période de l'étude, plusieurs cycles de mesures journalières de potentiel hydrique et de conductance stomatique ont été réalisés lors de journées ensoleillées.

\section{RÉSULTATS}

\section{Évolution journalière des paramètres hydriques}

Nous avons représenté fig 2 l'évolution journalière des densités de flux de sève, conductances stomatiques et potentiels hydriques pour 2 arbres du traitement sec (1 dominant et 1 dominé) et pour 1 arbre témoin bien alimenté en eau (dominant), lors de 3 journées caractéristiques pendant le desséchement : le jour 202 (20 juil) au début de la sécheresse, le jour 222 (9 août) lors de la phase de diminution rapide de 

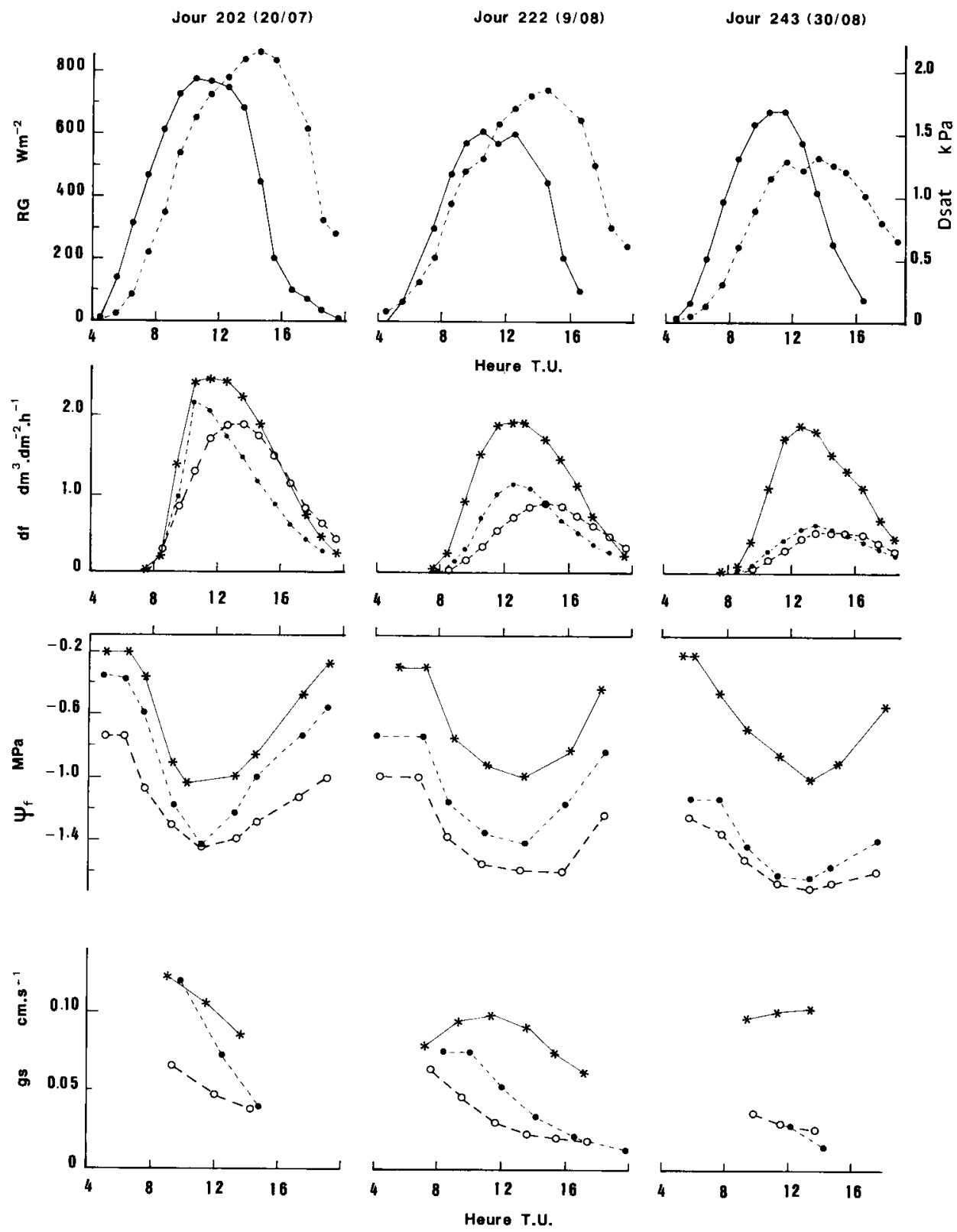

Fig 2 Variation journalière du rayonnement global indident $R G(-)$, du déficit de saturation de l'air Dsat $(--)$; de la densité de flux de sève $d f$, du potentiel hydrique foliaire $\psi f$ et de la conductance stomatique

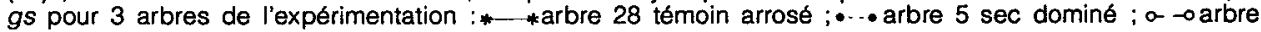
14 sec dominant. 
la transpiration et du potentiel hydrique et le jour 243 (30 août) lorsque la sécheresse était la plus intense.

La densité de flux de sève de l'arbre témoin présente une évolution journalière intermédiaire entre celle du rayonnement global et celle du déficit de saturation de l'air ; le maximum se situe à des valeurs proches de $2 \mathrm{dm}^{-3} \cdot \mathrm{dm}^{-2} \mathrm{~h}^{-}$ 1 , entre $12 \mathrm{~h}$. et $13 \mathrm{~h}$. TU.

On observe une diminution de la densité de flux de sève des 2 arbres du traitement sec, qui passe d'environ $2 \mathrm{dm} \cdot \mathrm{h}^{-1}$ (20 juil) à $1 \mathrm{dm} \cdot \mathrm{h}^{-1}$ (9 août) puis à $0,5 \mathrm{dm}^{3} \cdot \mathrm{dm}^{-2} \cdot \mathrm{h}^{-1}$ (30 août). On peut noter en outre :

- une augmentation plus tardive de la densite de flux des arbres secs par rapport au témoin en début de matinée :

- un déphasage des courbes de densité de flux de sève pour les arbres secs par rapport aux humides, qui va en augmentant, pour atteindre environ $2 \mathrm{~h}$ au plus fort de la sécheresse.

On observe, d'autre part, une évolution differente entre l'arbre dominant (14) et le domine (5) du traitement sec : densité de flux de l'arbre 14 sensiblement supérieure à celle de l'arbre 5 au début du dessèchement, puis une tendance inverse au jour 222, et niveaux comparables pour les 2 arbres en fin de dessèchement, (environ $25 \%$ celui de l'arbre témoin).

Pour les 3 journées, le potentiel de base de l'arbre témoin reste compris entre $-0,2$ et $-0,3 \mathrm{MPa}$, indiquant un état hydrique satisfaisant. Ce potentiel hydrique décroît dans la matinée pour atteindre un niveau proche de $-1,0$ $\mathrm{MPa}$. Les 2 arbres du traitement sec montrent une décroissance progressive du potentiel de base, qui passe de $-0,35$ à $-0,53$ puis à $-1,12 \mathrm{MPa}$ pour les 3 journées étudiées chez l'arbre dominé et $-0,74,-1,00$ et $-1,25 \mathrm{MPa}$ chez l'arbre dominant. Celui-ci montre ainsi un niveau de stress hydrique plus important que chez l'arbre dominé. On note enfin des valeurs minimales du potentiel de plus en plus basses et tardives.

Toutes ces observations montrent un couplage entre les variations diurnes de densite de flux et de potentiel hydrique ; ces relations seront examinées plus loin ;

En ce qui concerne l'évolution de la conductance stomatique ( $g s$ ), la même hiérarchie entre les 3 arbres apparaît une nouvelle fois:

- la conductance stomatique de l'arbre témoin est à toute heure supérieure à celle des 2 autres arbres; les valeurs maximales sont de l'ordre de 0,10 à $0,12 \mathrm{~cm} \cdot \mathrm{s}^{-1}$. L'allure des variations journalières n'est pas la même pour les 3 journées : le 20 juil, on constate une décroissance continue de gs, alors que le 9 août, gs présente un maximum vers $12 \mathrm{~h}$ TU ;

- l'arbre dominant du traitement sec présente les niveaux de gs les plus faibles, pour les jours 202 et 222, notamment pendant la matinée :

- l'arbre dominé du traitement sec se situe à un niveau intermédiaire, et rejoint l'arbre dominant en fin de journée, lorsque la régulation stomatique est la plus intense $\left(g s=0,04 \mathrm{~cm} \cdot \mathrm{s}^{-1}\right.$ le jour 202 et $0,02 \mathrm{~cm} \cdot \mathrm{s}^{-1}$ le jour 222). À la fin de la période de déssèchement (jour 243), ces 2 arbres présentent les mêmes niveaux de conductance stomatique, très faible dès le matin.

\section{Évolution saisonnière}

La figure 3 présente l'évolution au cours de l'expérimentation, des potentiels hydriques foliaires, de la transpiration relative des arbres du traitement sec (TS) par rapport à ceux de I'hu- 
D A T E
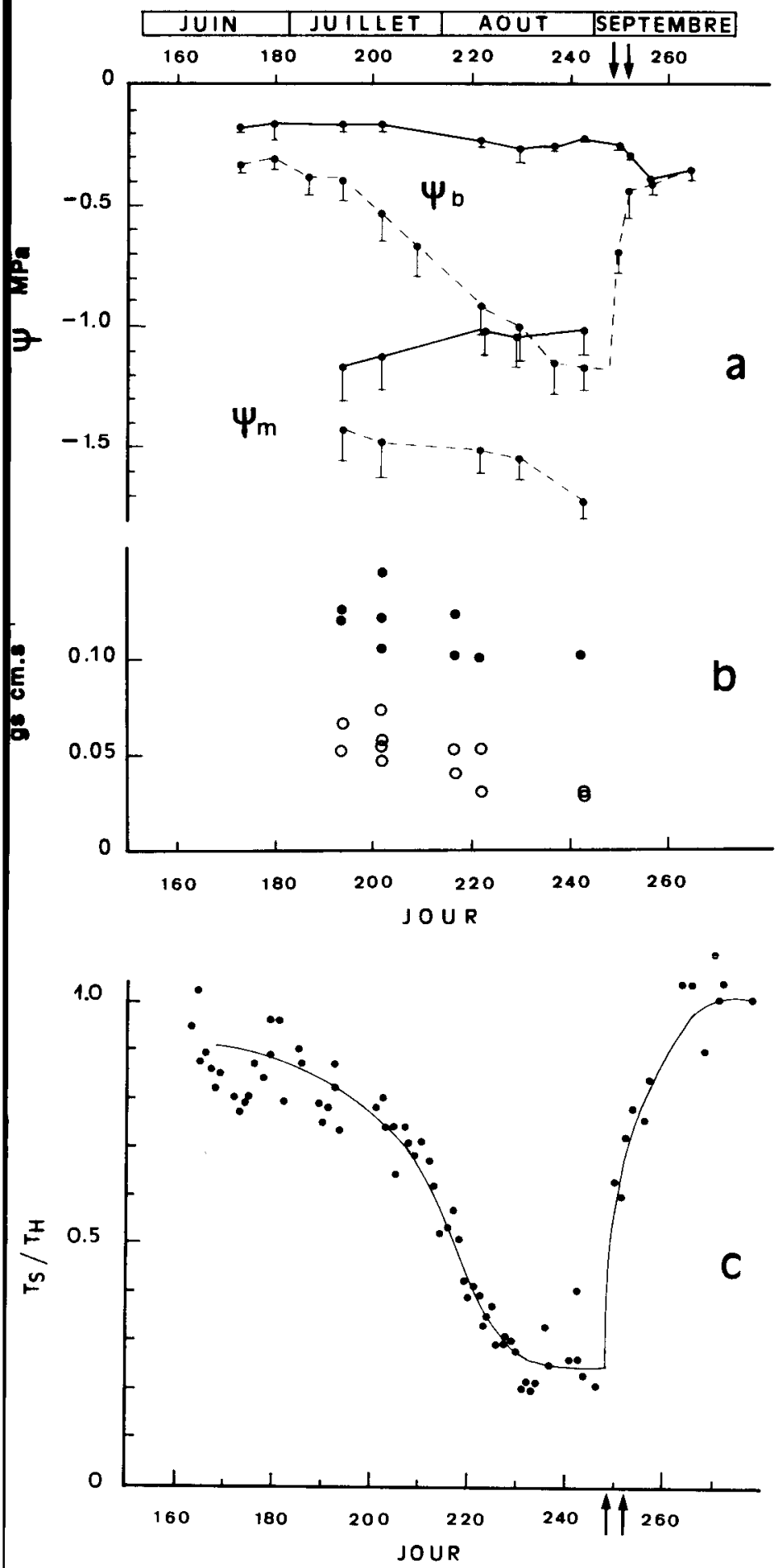

Fig 3a Évolution des potentiels hydriques de base $(\Psi b)$ et minimum $(\Psi \mathrm{m})$ pour les arbres des traitements humide $(-)$ et sec $(--)$. Les barres verticales indiquent les écarts types, les flèches les dates de l'irrigation des arbres du traitement sec. b Évolution de la conductance stomatique $g s$, mesurée entre $12 \mathrm{~h}$ ef $13 \mathrm{~h}$, des arbres des traitements humide ( $\bullet$ ) et $\sec (0)$. c Évolution de la transpiration relative TS/TH des arbres du traitement sec par rapport aux humides. 
mide $(T H)$, ainsi que de la conductance stomatique mesurée entre $12 \mathrm{~h}$ et $13 \mathrm{~h}$ TU. Les valeurs présentées ici sont des moyennes sur les 4 arbres de $T S$ et les 3 de $T H$. Dès le début de l'expérimentation, il semble que la transpiration relative des arbres de TS soit inférieure a 1 , indiquant un fonctionnement hydrique déjà affecté par la sécheresse. Ce rapport diminue lentement jusqu'à la fin du mois de juillet, puis plus rapidement pour atteindre environ 0,25 à la mi-août et s'y maintenir du 17 au 31 août.

La conductance stomatique (gs) n'a été mesurée de façon satisfaisante qu'à partir du jour 194 (12 juillet) ; à cette date, les arbres du traitement sec montrent des valeurs de $g s$ de l'ordre de $50 \%$ plus faible que celles des témoins $\left(0,12 \mathrm{~cm} \cdot \mathrm{s}^{-1}\right.$ pour TH contre 0,06 $\mathrm{cm} \cdot \mathrm{s}^{-1}$ pour TS) ; cet ecart est statistiquement significatif. Lorsque la sécheresse augmente, gs diminue encore, pour atteindre environ $0,03 \mathrm{~cm} \cdot \mathrm{s}^{-1}$ au jour 243 , soit $30 \%$ de la conductance des arbres témoins. II faut signaler que l'on observe pour les arbres du traitement humide, une diminution sensible de gs, en relation peut être avec celle de leur potentiel de base, malgré des apports d'eau (pluie et irrigation) fréquents.

Le potentiel de base $(\Psi b)$ moyen évolue de façon similaire ; au début des mesures $\Psi b$ des arbres de $T S(-0,33 \mathrm{MPa})$ est significativement inférieur à celui des arbres de $T H(-0,18 \mathrm{MPa})$. La diminution de $\Psi b$ des arbres desséchés est continue jusqu'au jour 237 (le 24 août), puis se stabilise à une valeur proche de $-1,2 \mathrm{MPa}$ jusqu'au moment de la réhydratation.

Le potentiel minimum $(\Psi m)$ (mesuré entre $14 \mathrm{~h}$ et $15 \mathrm{~h}$ TU) représenté sur la même figure ne montre par contre qu'une très faible diminution, de $-1,43$ $\mathrm{MPa}$ le 12 juil à $-1,73 \mathrm{MPa}$ le 30 juil.
Dès le début des mesures, les $\psi m$ des arbres secs sont toutefois significativement inférieurs à ceux des humides ( $\psi m$ compris entre $-1,0$ et $-1,2 \mathrm{MPa}$ ). L'examen des variations individuelles du $\psi b$ des arbres secs montre dès la $3^{\theta}$ mesure (le 5 juil) et jusqu'à la fin du dessèchement, que le potentiel de base des 2 plus petits arbres reste supérieur à celui des 2 plus gros, indiquant un meilleur état hydrique chez les premiers. Les mesures du 17 août en particulier ont été reportées pour chacun des 4 arbres dans le tableau II.

Tableau II. Valeurs du potentiel de base des arbres du traitement sec mesurées le 17 août (j 230).

\begin{tabular}{rcc}
\hline $\begin{array}{c}\text { Arbre } \\
\left(n^{\circ}\right)\end{array}$ & $\begin{array}{c}\text { Circonference (cm) } \\
\text { a } 1,3 \mathrm{~m} \text { du sol }\end{array}$ & $\Psi b(\mathrm{MPa})$ \\
\hline 5 & 30,0 & $-0,83$ \\
4 & 37,8 & $-0,91$ \\
14 & 51,9 & $-1,10$ \\
15 & 55,0 & $-1,15$ \\
\hline
\end{tabular}

Le potentiel de base apparaît d'autant plus faible que les arbres sont plus gros. Les 2 premiers arbres (4 et 5) sont des individus dominés, et il est probable que leurs houppiers sont soumis à une demande climatique moindre que ceux des dominants (14 et 15). D'ailleurs, en étudiant la densité de flux de sève (= flux par unité de surface de bois d'aubier) des arbres 4 et 5 par rapport à celle des arbres 14 et 15, on a constaté que :

- du début des mesures au jour 202, les arbres dominants présentaient un taux de transpiration supérieur à celui des arbres dominés, 
- du j 202 au j 244, le phénomène s'est inversé et les arbres dominés qui étaient dans un état hydrique plus favorable, avaient des densités de flux plus élevées que les arbres dominants, - après la réhydratation, la transpiration des arbres dominants est redevenue plus intense.

La réhumectation des arbres du traitement sec a été effectuée au moyen de 2 apports d'eau : 1200 I (soit $75 \mathrm{~mm}$ ) le 5 sept et 640 l $(40 \mathrm{~mm})$ le 8 sept. On peut voir, figure 2, que les arbres réhydratés ont amélioré alors progressivement leur fonctionnement hydrique. Leur transpiration est redevenue comparable à celle des arbres du traitement humide au bout de $20 \mathrm{j}$ environ, tandis que la remontée du potentiel de base a été plus rapide, la différence entre les 2 traitements s'annulant $7 j$ seulement après le premier apport d'eau.

\section{Conductance hydraulique}

Les calculs de conductance hydraulique spécifique ont été effectués pour chacun des 7 arbres du dispositif expérimental, au moyen d'une régression linéaire entre les densités de flux de sève et les potentiels hydriques foliaires. La figure 4 donne un exemple des relations experimentales obtenues, lors des journées étudiées pour un arbre du traitement sec, comparé à un arbre humide, et montre clairement la diminution progressive de la pente de la régression linéaire, indiquant celle de la conductance hydraulique au fur et à mesure que la sécheresse augmente. Le tableau III reporte les valeurs obtenues pour les 3 journées étudiees. On observe dès le jour 202 des valeurs de conductances supérieures pour chacun des arbres témoins à celles des arbres du traitement sec. La pro-

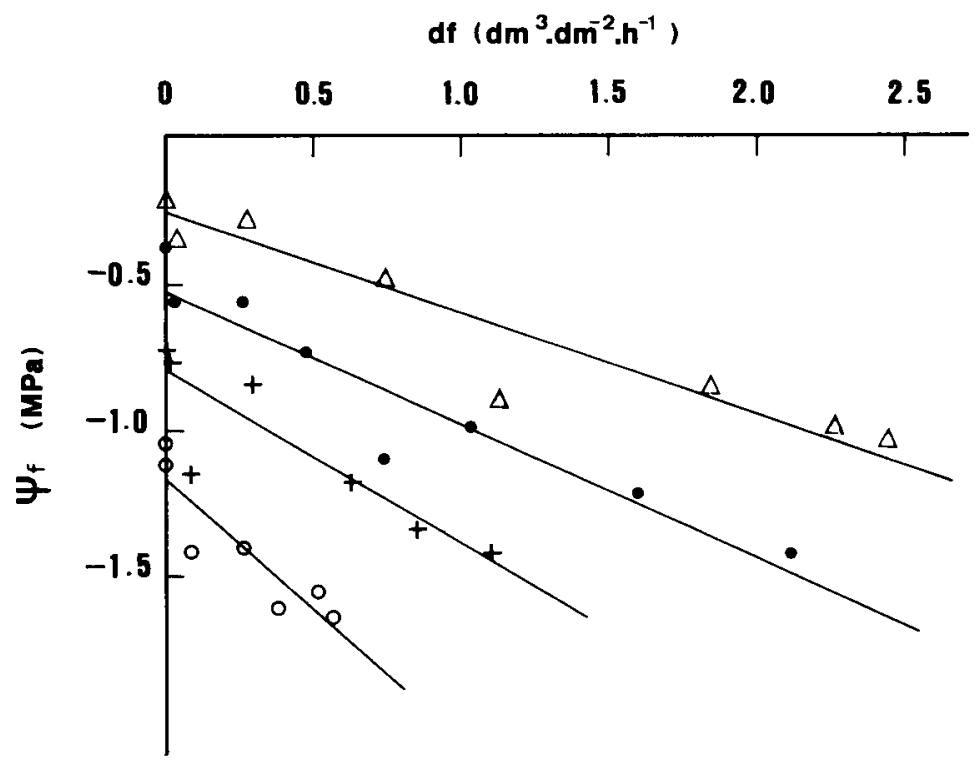

Fig 4 Relations entre le potentiel hydrique foliaire $\psi f$ et la densité de flux de sève $d f$, pour un arbre du traitement sec, les jours $202(\bullet), 222(+)$ et $243(\circ)$ et un arbre du traitement humide, le jour $202(\Delta)$. 
Tableau III. Évolution de la conductance hydraulique spécifique (exprimée en $10^{-5} \mathrm{~mol} \cdot \mathrm{m}^{-2} \cdot \mathrm{s}^{-1} \cdot \mathrm{Pa}^{-1}$ ) des 7 arbres, pour 3 journées à sécheresse croissante. Les carrés des coefficients de corrélation $r^{2}$ des régressions entre les potentiels hydriques foliaires et les densités de flux sont indiqués entre parenthèses. La lettre $n$ placée après $r^{2}$ indique une régression linéaire non significative au seuil de $5 \%$.

\begin{tabular}{lccccccc}
\hline Traitement & $\begin{array}{c}\text { Arbre } \\
\left(n^{\circ}\right)\end{array}$ & \multicolumn{2}{c}{ Jour 202} & \multicolumn{2}{c}{ Jour 222} & \multicolumn{2}{c}{ Jour 243} \\
\hline Humide & 28 & 0,407 & $(0,90)$ & 0,374 & $(0,85)$ & 0,383 & $(0,83)$ \\
& 19 & 0,439 & $(0,88)$ & 0,388 & $(0,90)$ & 0,347 & $(0,78)$ \\
& 29 & 0,349 & $(0,89)$ & 0,344 & $(0,68)$ & 0,300 & $(0,55) n$ \\
& moy & $\mathbf{0 , 3 9 8}$ & & $\mathbf{0 , 3 6 9}$ & & $\mathbf{0 , 3 4 3}$ & \\
Sec & 14 & 0,330 & $(0,74)$ & 0,186 & $(0,63)$ & 0,148 & $(0,75)$ \\
& 15 & 0,199 & $(0,60)$ & 0,122 & $(0,48) n$ & 0,063 & $(0,50) n$ \\
& 4 & 0,220 & $(0,70)$ & 0,133 & $(0,60)$ & 0,073 & $(0,71)$ \\
& 5 & 0,291 & $(0,87)$ & 0,210 & $(0,76)$ & 0,135 & $(0,83)$ \\
& moy & $\mathbf{0 , 2 6 5}$ & & $\mathbf{0 , 1 6 3}$ & & $\mathbf{0 , 1 0 5}$ & \\
\hline
\end{tabular}

$$
C \times 10^{5} \mathrm{~mol} \mathrm{~m}^{-2} \mathrm{~s}^{-1} \mathrm{~Pa}^{-1}(\mathrm{~L})
$$
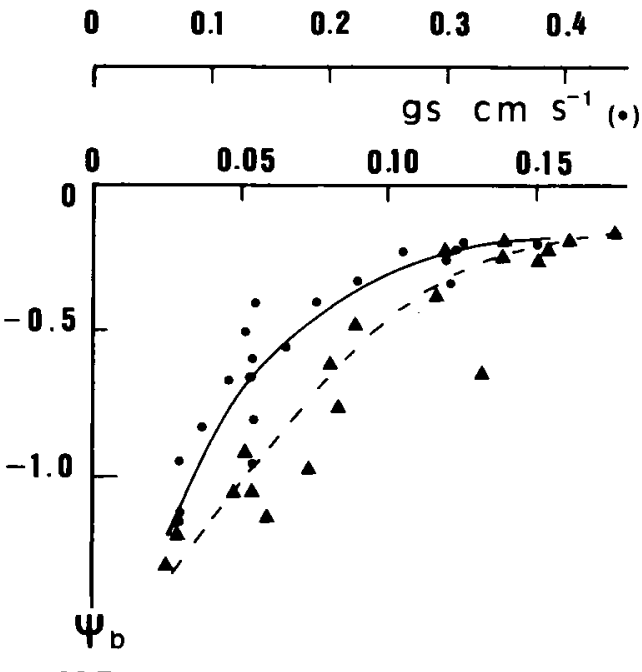

$\mathrm{MPa}$

Fig 5 Évolution de la conductance hydraulique spécifique $C(\Delta)$ et de la conductance stomatique gs mesurée entre $12 \mathrm{~h}$ et $13 \mathrm{~h}(\bullet)$ en fonction du potentiel de base $\psi b$.

gression de la sécheresse amène une nette décroissance de ce paramètre, les valeurs moyennes passant de $0,265 \cdot 10^{-5}$ à $0,163 \cdot 10^{-5}$ puis à
$0,105 \cdot 10^{-5} \mathrm{~mol} \cdot \mathrm{m}^{-2} \cdot \mathrm{s}^{-1} \cdot \mathrm{Pa}^{-1}$ pour des potentiels de base moyens égaux à $-0,53,-0,92$ et $-1,17 \mathrm{MPa}$ respectivement. L'évolution de la conductance hydraulique spécifique apparaît très liée à celle du potentiel hydrique de base, comme le montre la figure 5, pour l'ensemble des arbres étudiés. II faut signaler aussi une légère tendance à la diminution de la conductance hydraulique chez les arbres témoins, voisine de $14 \%$ entre les jours 202 et 243.

\section{DISCUSSION}

La contrainte hydrique appliquée sur les arbres du traitement sec peut être qualifiée de moyenne, comme l'atteste la valeur minimale atteinte du potentiel de base : -1,2 MPa. L'évolution de potentiel de base des arbres du traitement sec est comparable à celle observée par Aussenac et al (1984) sur le Douglas au moyen d'un dispositif expérimental similaire. La sécheresse a entraîné une diminution simultanée des flux et potentiels hydriques, et la mise en place précoce des 2 facteurs principaux de la régulation des flux d'eau 
par les arbres : conductance stomatique pour les échanges en phase vapeur et conductance hydraulique en phase liquide (du sol à la feuille). S'il est maintenant bien établi que la sécheresse édaphique provoque une diminution de la conductance stomatique (Schulze et al, 1987) via un effecteur encore hypothétique provenant du système racinaire, la diminution précoce de la conductance hydraulique (lorsque $\Psi b$ devient inférieur à $-0,3 \mathrm{MPa}$ ) pose plus de problèmes d'interprétation. II est difficile de n'attribuer cette variation qu'à des phénomènes de cavitation se produisant dans le système conducteur des feuilles, pour une gamme de potentiels hydriques foliaires, relativement élevés, donc a fortiori, dans les branches et le tronc. Une augmentation de la résistance au transfert de l'eau à l'interface sol-racine est une hypothèse probable, bien que notre expérimentation n'ait pu en préciser la localisation, et notamment s'il s'agit d'une élévation de la résistance dans le sol au voisinage des racines, ou une

\section{$\Psi_{b} \mathrm{MPa}$}

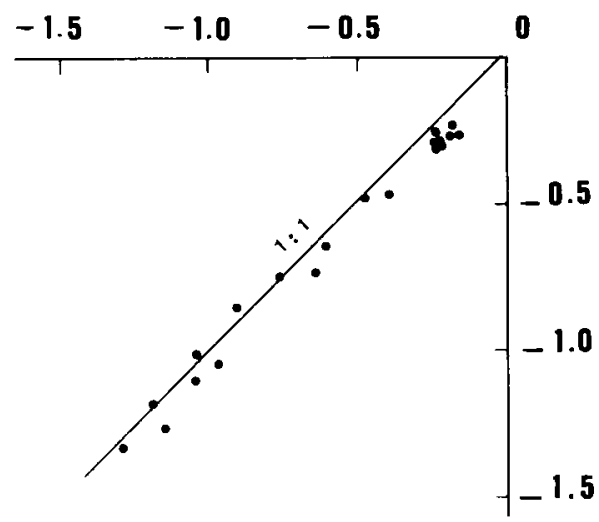

Fig 6 Relation entre l'ordonnée à l'origine des droites de régression $\psi f(d f)$ et le potentiel de base des 7 arbres étudiés, pour les jours 202, 222 et 243 diminution de la perméabilité des racines elles-mêmes. Pour Nnyamah et al (1978), toutefois la résistance au transfert de l'eau du sol à la racine reste très faible, voire négligeable, pour un potentiel hydrique dans le sol atteignant $-1,1 \mathrm{MPa}$; selon ces auteurs, la résistance à la circulation de l'eau dans la racine serait une composante prépondérante de la résistance hydraulique totale sur le trajet sol-feuille. Des mesures similaires réalisées sur Picea abies (Granier et al, 1988) ont aussi montré un abaissement précoce et important de la conductance hydraulique spécifique pour un potentiel de base encore élevé $(-0,5 \mathrm{MPa})$. Cohen et al (1983), sur Citrus sinensis ne trouvent pas, au contraire, de diminution significative de conductance hydraulique, jusqu'à un potentiel de base égal à 1,6 $\mathrm{MPa}$, ce qui peut laisser supposer un comportement lié à l'espèce.

En ce qui concerne la méthode utilisée pour évaluer la conductance hydraulique, la prise en compte du potentiel de base, au lieu du potentiel hydrique du sol, ne semble pas introduire de biais dans nos résultats. En particulier, si l'on compare les ordonnées à l'origine des régressions entre $\psi f$ et la densité de flux des 7 arbres pour les 3 journées étudiées avec les potentiels de base mesurés, on constate (fig 6) que ces valeurs sont très proches. Il existe ainsi une bonne symétrie des points autour de la droite qui passe par $\psi b$, et de pente $-1 / C$ (équation (2)), et on peut admettre que le potentiel de base représente bien la valeur du potentiel hydrique à l'interface sol/racine pour un flux nul.

Les arbres bien alimentés en eau ont montré une diminution faible, mais perceptible de conductance, aussi bien stomatique qu'hydraulique, en relation encore une fois, avec la décrois- 
sance de leur potentiel de base, bien que le sol ait été maintenu à un niveau proche de la capacité au champ. II pourrait s'agir d'un phénomène saisonnier, impliquant des mécanismes de la croissance, tant de système racinaire que de l'appareil aérien.

Le comportement respectif des arbres dominants et dominés en condition de stress hydrique est à relier aux conditions de milieu et à des potentialités de fonctionnement hydrique différentes. Sur épicéa, Breda (1987) a pu mette en évidence une répartition analogue des potentiels de base dans une population d'arbres soumis à la sécheresse.

\section{RÉFÉRENCES}

Aussenac G, Granier A, Ibrahim M (1984) Influence du dessèchement du sol sur le fonctionnement hydrique et la croissance du Douglas (Pseudotsuga menziesii (Mirb) Franco). Oecol Plant, 5, 19, 241253

Breda N (1987) Caractérisation du fonctionnement hydrique d'épicéas en conditions naturelles : utilisation d'une méthode de mesure du flux de sève brute. Mémoire de Maîtrise, Université de Nancy I

Cohen Y, Fuchs M, Cohen S (1983) Résistance to water uptake in a mature citrus tree J Exp Bot, 34, 451-460

Colin F (1988) Modifications du fonctionnement hydrique lors d'une sécheresse édaphique. Cas de Abies bornmulleriana. Rapport de DEA Université Nancy I, $54 \mathrm{p}$
Granier A (1985) Une nouvelle méthode pour la mesure du flux de sève brute dans le tronc des arbres. Ann Sci For, 42, 81-88

Granier A (1987) Mesure du flux de sève brute dans le tronc du Douglas par une nouvelle méthode thermique. Ann Sci For, 44, 1-14

Granier A, Breda N, Claustres JP, Colin F (1988) Variation of hydraulic conductance of some adult conifer in natural conditions. Poster présenté au Symp Int Physiol Arbres For, Nancy, 26-30 septembre 1988

Grier C C, Waring RH (1974) Conifer foliage mass related to sapwood area. For Sci, 20, 205-206

Landsberg JJ, Blanchard TW, Warrit B, (1976) Studies on the movement of water through apple trees. $J$ Exp Bot, 27, 579596

Levy G, Becker M (1987) Le dépérissement du sapin dans les Vosges : rôle primordial de déficits d'alimentation en eau. Ann Sci For, 44, 403-416

Nnyamah JV, Black TA, Tan CS (1978) Resistance to water uptake in a Douglas fir forest. Soil Sci, 126, 63-76

Schulze ED, Cermak J, Matyssek R, Penka $M$, Zimmermann $R$, Vasicek $F$, Gries $W$, Kucera J (1985) Canopy transpiration and water fluxes in the xylem of the trunk of Larix and Picea trees. A comparison of xylem flow, porometer and cuvette measurements. Oecologia, 66, 475-483

Schulze ED, Turner NC, Gollan T, Shackel KA, (1987) Stomatal responses to air humidity and soil drought. In : Stomatal Function (Zieger, E, Farquhar GD, Cowan IR eds) Standford University press

Whitehead D (1978) The estimation of foliage area from sapwood basal area in scots pine. Forestry (Oxf) 51, 137-149 